Research Article

\title{
Mucoadhesive Particles: A Novel, Prolonged-Release Nanocarrier of Sitagliptin for the Treatment of Diabetics
}

\author{
Nagaraja SreeHarsha $\mathbb{D}^{1,},{ }^{1,2}$ Chandramouli Ramnarayanan $\left(\mathbb{D},{ }^{3}\right.$ Bandar E. Al-Dhubiab $(\mathbb{D}){ }^{1}$ \\ Anroop B. Nair $\mathbb{D}^{1}{ }^{1}$ Jagadeesh G. Hiremath, ${ }^{2}$ Katharigatta N. Venugopala $\mathbb{D}^{1,4}$ \\ Roopashree T. Satish $\mathbb{D},{ }^{5}$ Mahesh Attimarad $\mathbb{D},{ }^{1}$ and Arshia Shariff $\mathbb{D}^{6}$
}

${ }^{1}$ Department of Pharmaceutical Sciences, College of Clinical Pharmacy, King Faisal University, Al-Ahsa, Saudi Arabia

${ }^{2}$ Department of Pharmaceutics, Vidya Siri College of Pharmacy, Bengaluru, India

${ }^{3}$ Department of Quality Assurance, Krupanidhi College of Pharmacy, Bengaluru, India

${ }^{4}$ Department of Biotechnology and Food Technology, Durban University of Technology, Durban 4001, South Africa

${ }^{5}$ Department of Pharmacognosy, Government College of Pharmacy, Bengaluru, India

${ }^{6}$ Department of Pharmaceutics, Alard College of Pharmacy, Savitribai Phule, Pune University, Pune, India

Correspondence should be addressed to Nagaraja SreeHarsha; sharsha@kfu.edu.sa

Received 17 May 2019; Revised 21 August 2019; Accepted 19 September 2019; Published 3 November 2019

Academic Editor: Wan-Liang Lu

Copyright (C) 2019 Nagaraja SreeHarsha et al. This is an open access article distributed under the Creative Commons Attribution License, which permits unrestricted use, distribution, and reproduction in any medium, provided the original work is properly cited.

\begin{abstract}
Sitagliptin (MK-0431) is a widely and commonly used oral hypoglycemic drug in the treatment of type 2 diabetes mellitus; patients typically take higher doses of this drug $(50 \mathrm{mg}$, twice daily). One drawback is that only $38 \%$ of the drug is bound reversibly to plasma proteins and $79 \%$ is excreted in urine without being metabolized. To overcome this issue, there is a need for a better drug-delivery method to improve its efficacy in patients. It has been found that in existing formulations, the drug content is $72.5 \% \pm 5 \%$ and the percentage yield is $84.9 \% \pm 3 \%$. In this study, sitagliptin nanoparticles (sizes ranging from 210 to $618 \mathrm{~nm}$ ) were developed. The bioadhesion properties of the nanoparticles, as well as the swelling of the nanoparticles on the mucus membrane aided in sustained drug release. The pattern of drug release was in accordance with the Peppas model. Fourier-transform infrared (FTIR) spectroscopy demonstrated that there were no significant interactions between sitagliptin and chitosan. Differential scanning calorimetry (DSC) results showed an absence of drug peaks due to the fact that the drug was present in an amorphous state. Mucoadhesive nanoparticles were formulated using sitagliptin and were effective for about 12 hours in the gastrointestinal tract. When compared to conventional sitagliptin administration, use of a nanoparticle delivery system demonstrated greater benefits for use in oral delivery applications. This is the first time that a drug-delivery method based on the mucoadhesive properties of nanoparticles could prolong the drug-release time of sitagliptin.
\end{abstract}

\section{Introduction}

Diabetes represents a complex set of health disorders that affect many patients irrespective of age. Type 2 diabetes mellitus (T2DM) is a form of diabetes that is characterized by the body's inability to produce insulin [1]. Given the widespread nature of this commonly occurring disease, researchers around the world have investigated and developed novel drug-delivery systems that aim to treat it.
The Kingdom of Saudi Arabia is a developing country populated by over 28 million people [2], of which $25 \%$ have been diagnosed with diabetes mellitus (DM) [3]. The prevalence of DM has been expected to increase to $40 \%-50 \%$ by 2020 [4]. Medications to treat DM are usually administered orally, and the side effects associated with existing treatments are increased body weight, hypoglycemia, and gastric intolerance. For this reason, T2DM patients remain insufficiently treated. Hence, there has been an immediate need to identify newer therapeutic options for T2DM [5]. 
Existing medications that were developed to manage DM are characterized by shorter half-lives, meaning that patients must take higher dosages per day, which is not good. Hence, drugs that have prolonged gastric residence times must be formulated to ensure sustained release, which can aid in the prolonged effects of the drug. For this reason, the drug should remain in the upper part of the gastrointestinal tract or in the stomach for longer periods of time. This can only be achieved by sustaining the drug's release until all of the drug is completely released over a desired period of time [6-8]. Existing drugs with prolonged gastric residence times can reveal greater insights into new therapeutic options. Drugs that float and deliver is a concept that was studied by Davis for the first time in 1968, whereby drugs that are bulky in density, and which are lower in density than gastric juice $\left(1.004 \mathrm{~g} / \mathrm{cm}^{3}\right)$, can remain in the gastrointestinal tract for longer periods, thus being bioavailable and lowering the elimination/degradation of the drug with localized action [9].

When examining the drugs that are available for treating T2DM, sitagliptin (SCN) remains among the best and most effective therapeutic options. In October 2006, the United States Food and Drug Administration (FDA) approved SCN as a basic inhibitor of DPP-4 in the treatment of T2DM. SCN thus falls under the class of DPP-4 inhibitors. It controls $\mathrm{HbA1c}$ and fasting glucose levels in patients with T2DM [10]. Many reports have indicated that SCN is a mucoadhesive microsphere with higher gastric retention times [11]. It has been formulated as bilayered tablets with simvastatin [12], trilayered tablets with metformin [13], and gastroretentive floating tablets [14].

DPP-4 inhibitors are keen on extending the actions of endogenous glucagon-like peptide (GLP)-1. The DPP-4 enzyme inhibits proteolysis, thus elevating GLP-1 levels by two to three times, consequently inhibiting $>90 \%$ of plasma DPP-4 activity in vivo over the course of 24 hours.

Sitagliptin (MK-0431) is involved in inhibiting or delaying incretin degradation. The main functions of DPP-4 inhibitors include mimicking incretin while stimulating insulin secretion, inhibiting glucagon secretion and apoptosis, and ultimately preserving $\beta$-cell mass. Patients must strictly adhere to dosage intervals of $50 \mathrm{mg}$ twice per day [15-17]. Recently, we have demonstrated the potential of albumin nanoparticles in enhancing a drug's mucoadhesive properties, thereby improving the efficiency of sitagliptin.

Polymeric nanoparticles can effectively alter the pharmacokinetics of drug molecules; they are currently considered the ideal formulations when developing treatments targeting chronic diseases, including diabetes [18]. Despite the fact that hydrogels exhibit highly mucoadhesive properties, they are neutral and weakly interact with the mucosal layer of the membrane $[2,4]$. However, cationic chitosans, which are copolymers of $\mathrm{N}$-glucosamine, exhibit unique physicochemical and biological properties. In contrast, chitosans, which are cationic, natural copolymers of $\mathrm{N}$-glucosamine, are produced by the deacetylation of chitin and have unique physiochemical and biological properties. Being highly biocompatible, biodegradable, and nontoxic, chitosans are unique to the development of mucoadhesive drug-delivery system (MDDS) and feature special properties $[5,6,19,20]$. Chitosan has been widely formulated into drug carriers, including nano- to microsized particles [20-22].

An ionic bond between the amino group of chitosans and the sialic acid of mucins can yield a formulation that is retained on the mucosal membrane where the drug is to be delivered. Therefore, a matrix formed by chitosan acts as a balanced drug-delivery system and sustains the drug release [15]. Hence, chitosan proved to be effective in the treatment of various diseases over a prolonged period of time by fixing the drug (with its enhanced mucoadhesive properties) at the site of application. In contrast to our earlier study, a comprehensive assessment was carried out to explore how best to enhance the therapeutic delivery of sitagliptin-loaded chitosan nanoparticles through prolonged retention at the site of absorption, thereby improving its clinical efficacy. Spray-drying was used to synthesize the nanoparticles, which delivered SCN orally, to treat patients with diabetes. Chitosan was used in the formulations to promote the bonding of the drug to the mucosal layer, so that a matrix could form and prolong the drug-release time.

\section{Materials and Methods}

Sitagliptin, chitosan, acetic acid sodium deoxycholate (purity $\geq 97 \%$ ), and membrane filters $0.22 \mu \mathrm{m}$ in size were purchased from Sigma-Aldrich Co. (St. Louis, MO, USA). The reagents used were of high-performance liquid chromatography (HPLC) grade. Double-distilled deionized water was used for the experiments.

2.1. Formulation of the SCN Nanoparticles. A total of $1,000 \mathrm{mg}$ each of SCN and chitosan were taken and combined in $250 \mathrm{~mL}$ of deionized water. The solutions were made to a $\mathrm{pH}$ level of 5 ; acetic acid $(0.25 \%$ [v/v]) was used to prepare the solution for spray drying. The spray-dried nanoparticles were developed using a spray dryer in a laboratory setting (Buchi B-90, Labortechnik AG, Flawil, Switzerland). The process set at an inlet temperature of $100^{\circ} \mathrm{C}$ with a rate of aspiration of $100 \%$, a spray flow rate of $160 \mathrm{~L} /$ hour, and the pump was adjusted to $2.5 \mathrm{~mL} /$ minute. These balanced the outlet temperature of $35^{\circ} \mathrm{C}$. A collecting vessel was used to collect the dried powders and the yield rate was recorded. All experiments were performed in triplicate.

2.2. Scanning Electron Microscope (SEM). The spray-dried microspheres were observed under a scanning electron microscope (EVO MA15, Zeiss, Oberkochen, Germany) at a high vacuum of $20 \mathrm{kV}$. The spray-dried nanoparticle samples were sputter-coated with an ultrathin layer of electrically conducting metal (gold) in a partial vacuum. Micrographs were observed, captured, and imaged.

2.3. Nanoparticle Size Measurement. The size of each particle was measured using a Malvern Zetasizer Nano ZS (Malvern Instruments, Malvern, UK). The particles were kept in 
absolute ethanol for dispersion (refractive index: -2.43 ) and were measured at room temperature.

2.4. Swelling Behavior Study. The swelling behavior of the spray-dried nanoparticles was carried out in simulated gastric fluid ( $\mathrm{pH}$ 1.2). The dried nanoparticles sizes were measured under a microscope after the nanoparticles were dipped in gastric fluid ( $\mathrm{pH} 1.2)$ at intervals of 5 minutes, 15 minutes, 30 minutes, 1 hour, 3 hours, 5 hours, 8 hours, and 12 hours. The percent swelling was calculated using the formula

$$
\text { Swell }[\%]=M t-\frac{M 0}{M 0} \times 100,
$$

where time $" t "=[M t] ;$ initial time $[t=0[M 0]]$.

2.5. Production Yield. The nanoparticles were collected from the spray-drying chamber and the total nanoparticle yield was measured. The following formula was used to calculate the yield:

$$
\% \text { yield }=\frac{[\text { actual yield }]}{[\text { theoretical yield }]} \times 100 \text {. }
$$

2.6. Wash-Off Test (In Vitro). The Ranga Rao and Buri [23] method was used to determine the adhesiveness of the nanoparticles. Rats were anesthetized using isoflurane $/ \mathrm{O}_{2}$. The mucosal layer of the stomach $(\sim 5 \mathrm{~cm}$ in length, $1 \mathrm{~m}$ in width) was neatly cut and washed in normal saline. Then, $100 \mathrm{mg}$ of the drug was carefully placed on the mucosal surface and allowed to interact for up 30 minutes [24]. The mucus layer was placed in a desiccator for up to 15 minutes; the humidity was set at $90 \%$. The mucosal layer was washed in saline at a flow rate of $5 \mathrm{~mL} /$ minute. The concentration of the drug was read in a spectrophotometer.

2.7. In Vitro Drug Release and Kinetics of the SCN Formulation. The in vitro drug release of the SCN formulation in the solution was studied sequentially at a $\mathrm{pH}$ of 1.2 and a $\mathrm{pH}$ of 6.8 to stimulate fasting $\mathrm{pH}$ levels in the stomach and intestine, respectively. The prepared nanoparticles were briefly suspended in the solution at a concentration of $0.1 \%$ $(\mathrm{w} / \mathrm{v})$; then, the solution was incubated in an orbital shaker at $37^{\circ} \mathrm{C}$ at $100 \mathrm{rpm}$. Following incubation for 30 minutes, 1 hour, and 2 hours at a pH of 1.2 and for 30 minutes, 1 hour, 2 hours, 4 hours, 8 hours, and 12 hours at a $\mathrm{pH}$ of 6.8 , a $100 \mu \mathrm{L}$ sample was aliquoted and adjusted with an equal volume of fresh medium accordingly. Each aliquot was then centrifuged (3,000 rpm for 15 minutes) in a plastic tube and the supernatant was separated to further determine the SCN released by ultraviolet (UV) spectrophotometer at $267 \mathrm{~nm}$. The obtained results were compared with different kinetic models to better understand the mechanism of drug release.

2.8. FTIR Spectra of Sitaglitpin, Chitosan, and the Formulation. The absorption spectra were obtained in the range of $500-4,000 \mathrm{~cm}^{-1}$ using an attenuated total reflectance (ATR) FTIR (8400, Shimadzu, Kyoto, Japan). Samples were put on the sample holder and the distance between the sample holder screw and zinc selenide crystal was adjusted to obtain 64 scans following a background correction.

2.9. DSC Analysis of Sitaglitpin, Chitosan, and the Formulation. A DSC analysis was performed using a differential scanning calorimeter (DSC 1, Mettler-Toledo, Columbus, $\mathrm{OH}, \mathrm{USA}$ ). The samples were sealed in standard aluminum pans and heated from $40^{\circ} \mathrm{C}$ to $300^{\circ} \mathrm{C}$ at a heating rate of $10 \mathrm{~K} /$ minute under nitrogen gas and purged at a $40-60 \mathrm{~mL} /$ minute flow rate.

2.10. Drug Distribution in the GIT. Male Sprague-Dawley rats weighing 250-300 g, and which were fasted for 20-24 hours before the experiment, were selected. The rats were divided into five groups of 15 rats. The control rats (the first group) were given the SCN solution orally. The second to fifth sets received the SCN nanoparticles. Orally administered nanoparticles were injected nonanesthetically using a rubber tube at a dilution of $25 \mathrm{mg}$ (nanoparticles) $/ 1 \mathrm{~mL}$ (saline). Three rats were sacrificed from each group following 1 hour, 3 hours, 5 hours, 8 hours, and 12 hours of administration. The stomach and small intestine were taken together (Section 1) and subdivided into six sections (Sections $2-7 ; 14 \mathrm{~cm}$ in length each). These were cut open, so that the mucosal layer would become exposed. The nanoparticles were collected using a spatula by neatly removing the layer. SCN was extracted by homogenization and the mashed solution was incubated for 24 hours; it was then spun at $1,000 \times \mathrm{g}$ for 30 minutes. The supernatant was spectrophotometrically analyzed.

\section{Results and Discussion}

The mucoadhesive SCN nanoparticles had structures that differed in terms of outer-layer texture and drug-loading patterns, which depended on different methods to create various formulations. The drug loading was around $72.5 \% \pm 5 \%$, whereas the nanoparticle content was $84.9 \% \pm 3 \%$ in $500 \mathrm{mg}$ of powder, which is relatively higher than the data reported in our earlier study [24]. Product was lost while loading the drug, as the nanoparticles were left behind in the spraying chamber or were lost during manual collection from the electrode [11].

The developed nanoparticles demonstrated an almost spherical scattering pattern, which occurred in a rhythmic manner. There was no evidence of any contractions on the surface (Figure 1(a)), and the nanoparticles were compared with the pure drug (Figure 1(b); sitagliptin) and polymer (Figure 1(c); chitosan). Uniform spray drying was observed for all spheres. The nanoparticles differed in shape due to the varied moisture content and the drying temperature used. Specifically, the smaller the particle, the faster it was able to dry. 


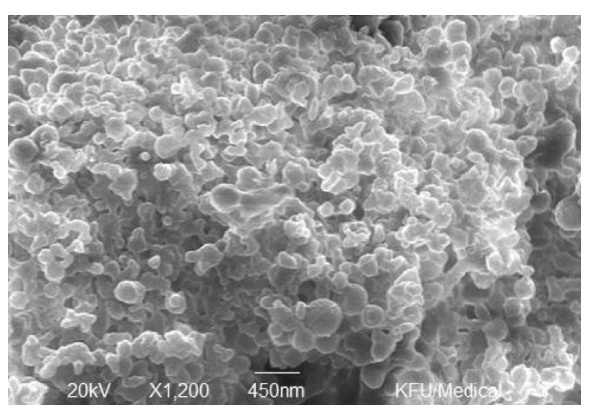

(a)

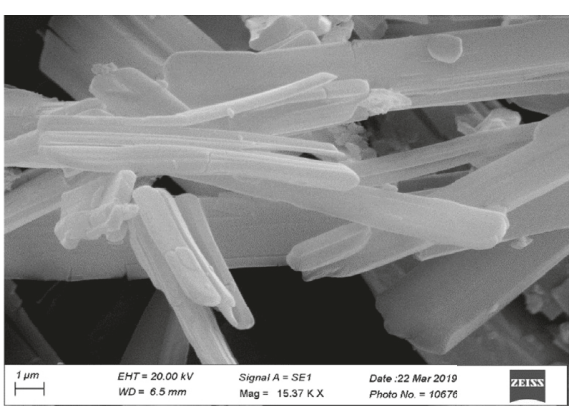

(b)

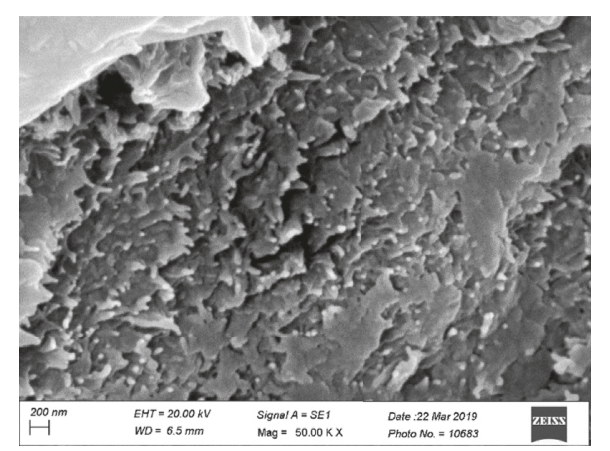

(c)

FIgURe 1: SEM image showing the formulation (a), pure drug (b), and the chitosan (c).

Our scanning electron microscopy (SEM) results varied in accordance with the size of the SCN nanoparticles (Figure 2), with a mean size of $350 \mathrm{~nm}$; smaller sizes were found to be $210 \mathrm{~nm}$ and larger sizes were $618 \mathrm{~nm}$. However, these data signify that the particle sizes are relatively small when compared with the data observed in our earlier study [24]. Typically, small particles will enhance the total surface area given their narrow size. Narrowing of the nanoparticle size indicated abrupt SCN release at the absorption site; thus, this was associated with the sustained release of a considerable amount of SCN and demonstrated the hypoglycemic effects of this drug.

The swelling percentage of the SCN nanoparticle formulation was also monitored at different time intervals (Figure 3). It was observed that all nanoparticles rapidly swelled within 7.2 minutes in saline at a $\mathrm{pH}$ level of 1.2 . The mucoadhesiveness of the nanoparticles was reported earlier, and the cohesiveness of the polymers was represented by the swelling index [25]. Overall, it was found that liquid in the stomach was absorbed by the nanoparticles. The capillary action of the mucosa contributes to stronger adhesion. SCN formulation swelling was around $168 \% \pm 15 \%$.

Bioadhesion studies were carried out to understand the nature of mucoadhesion. Our results were validated by the significant increase in SCN nanoparticles ex vivo (31.5 \pm 0.25 hours). This was due to the electrostatic bond between the nanoparticles and glycoprotein in mucin. The primary reaction observed was between the hydrogen bond, the electrostatic attraction of the nanoparticles, and the hydrogen atoms of mucin, thus resulting in strong adhesive forces among the nanoparticles. This mucoadhesion formed the basis for the systematic release of SCN from the nanoparticles into the GIT. SCN bursts directly from the intestinal mucosa in rats in the control group due to the absence of adhesiveness.

Different from our earlier study, the profiles of the SCN drug release formulations in vitro were assessed while maintaining the different solutions at $\mathrm{pH}$ levels of 1.2 and 6.8 for 2 hours and 12 hours at $37^{\circ} \mathrm{C}$, which are similar to the $\mathrm{pH}$ levels of the stomach and small intestine, respectively (Figure 4). There was an initial burst in the first hour due to the desorption of SCN at the top of the chitosan nanoparticles, followed by a delay in drug release. The amount of SCN released was found to be around $45.1 \%$ at 2 hours ( $\mathrm{pH} 1.2$ ), showing that the SCN was loaded deep inside the nanoparticles, facilitating the release of SCN into the stomach while fasting. Therefore, an initial burst favors the maintenance of a constant concentration of the therapeutic drug at $\mathrm{pH}$ 1.2. At $\mathrm{pH} 6.8$, the release of SCN was $98.1 \%$ over the next 12 hours. These results revealed the systematic and perfect release of SCN nanoparticles.

Our experimental results from this in vitro study were compared with different kinetic models, such as those of Baker and Lonsdale (first order); Weibull, Hixson, and Crowell; and Peppas and Higuchi $[24,26]$. Sigma Plot (Ver. 9.01) was used different mathematical models to determine the release mechanism. The results best fit with the Peppas model, with a correlation coefficient of $R^{2}=0.9928$. The fit with the Peppas model indicates that the release of SCN from the nanoparticles occurred by a polymer matrix and obeyed Fick's law of diffusion, which transported the drug from the polymeric matrix. The mucoadhesion of the nanoparticles, 


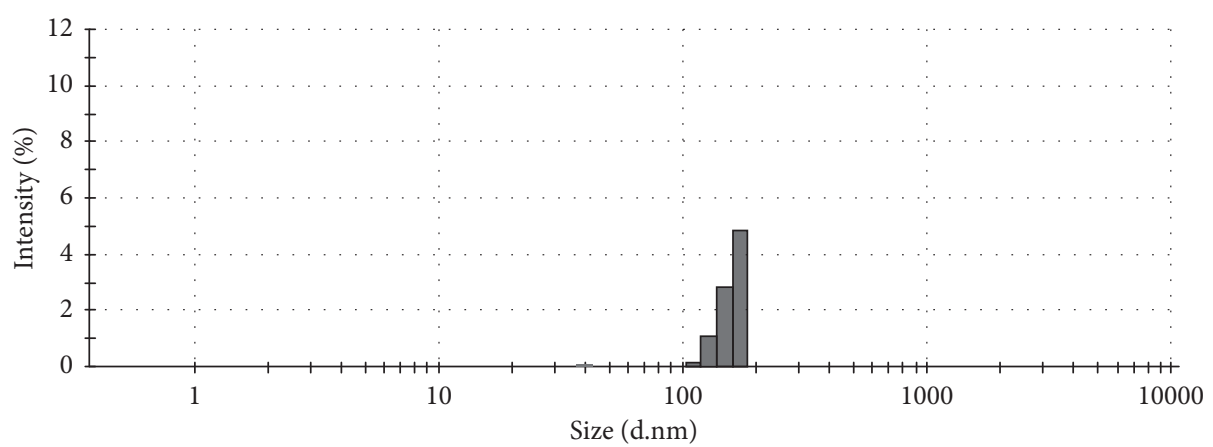

FIGURE 2: Nanoparticle size plotted against intensity (\%). The average size of the particles was $\sim 350 \mathrm{~nm}$.

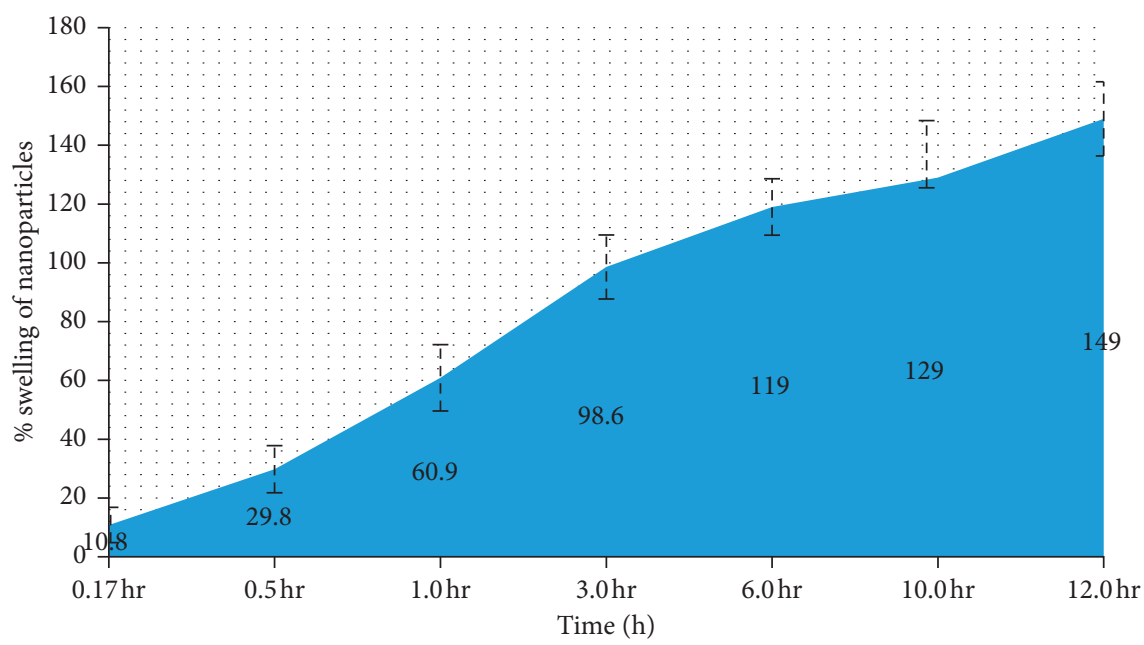

FIgURE 3: Swelling percentage of the nanoparticle formulation. Particle swelling began within 7 minutes, and a gradual increase was noticed given the prolonged time spent on the mucosal membrane.

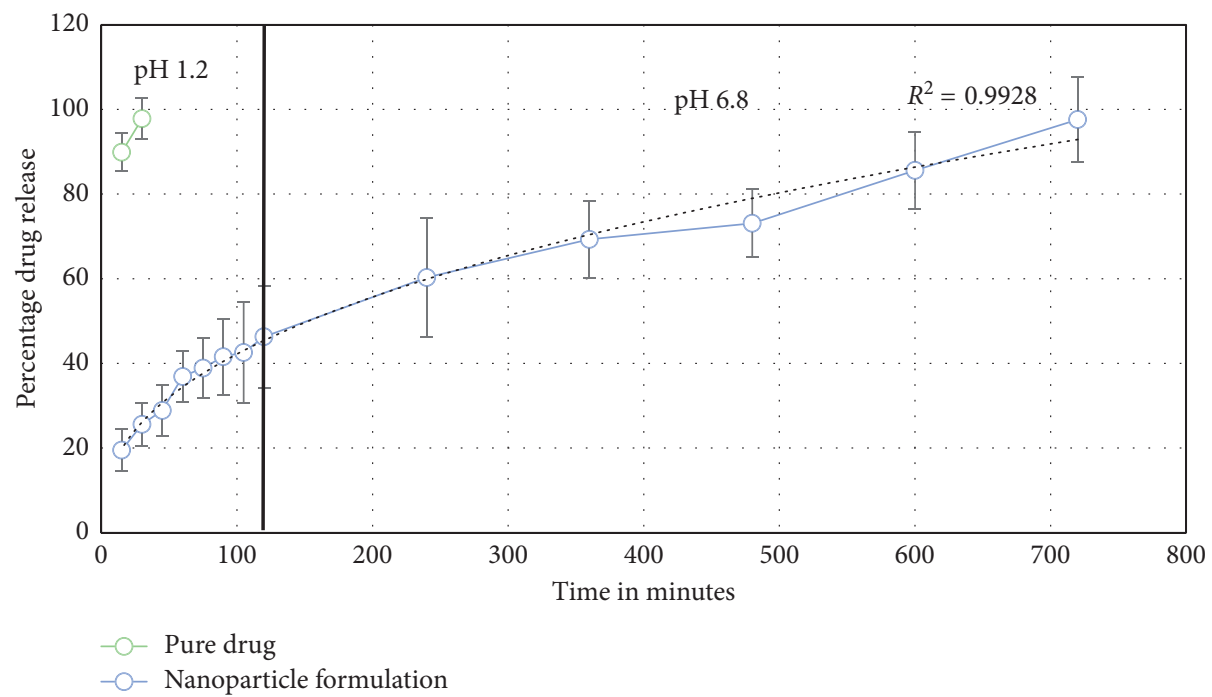

FIGURE 4: In vivo release comparisons in the stomachs of Sprague-Dawley rats following oral administration of the pure drug and nanoparticle formulation.

which was characterized by the initial swelling and adsorption of (or the weak binding to) the surface of polymeric nanoparticles quite likely contributed to the high rate of "burst" [27]. After this burst cascade, the controlled release of SCN from the nanoparticles was maintained for many hours in the stomach. 


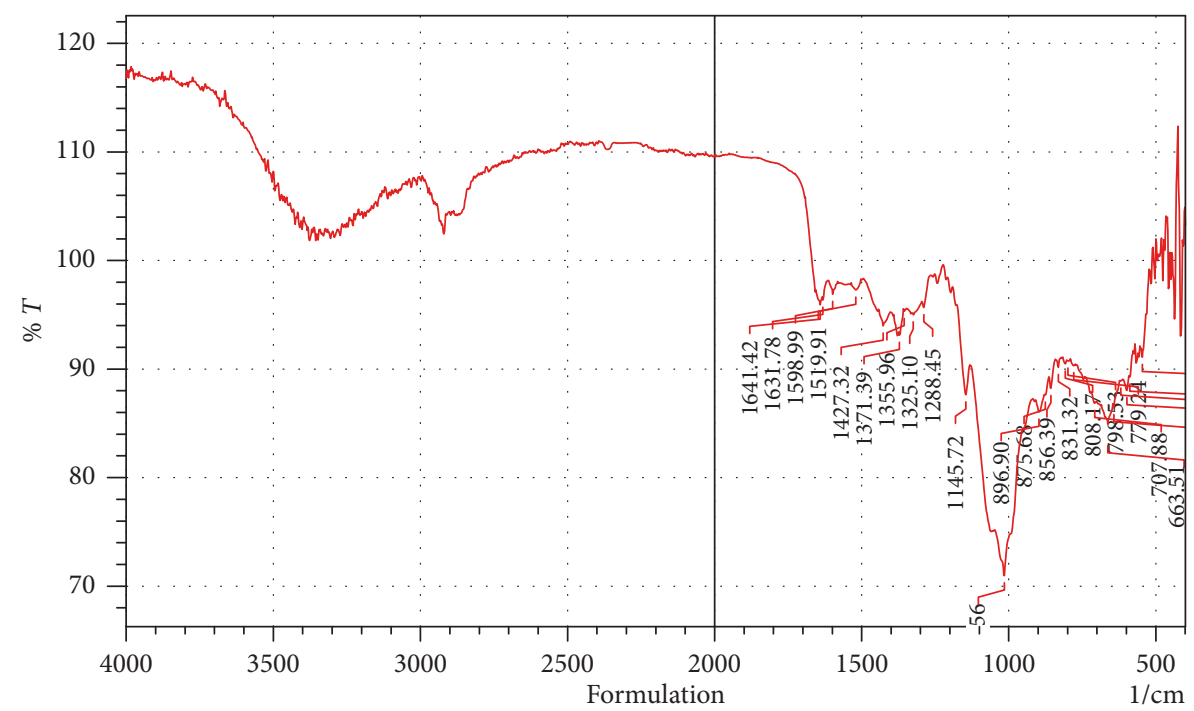

(a)

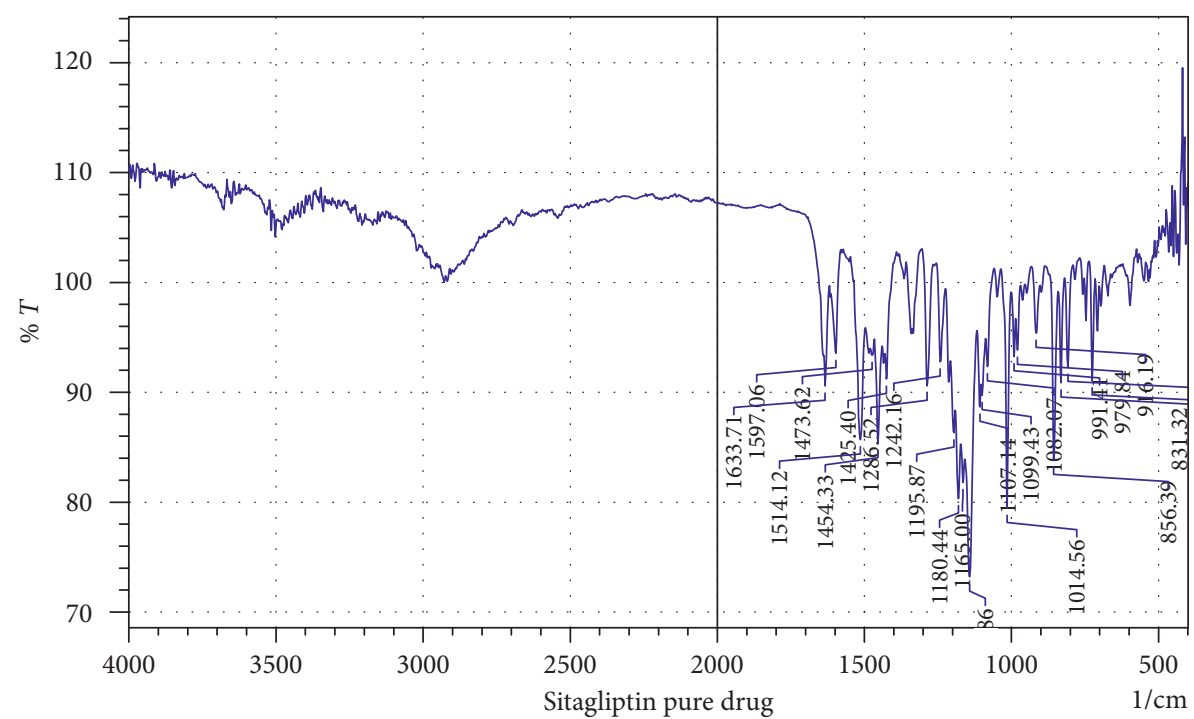

(b)

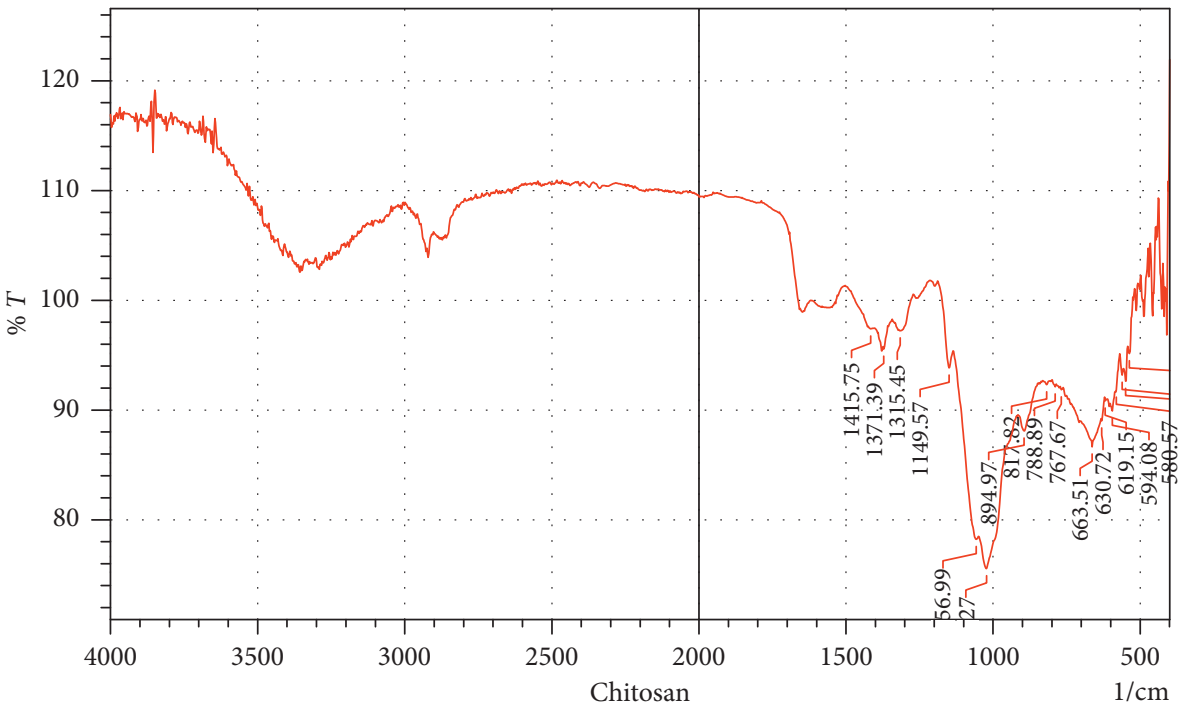

(c)

Figure 5: The FTIR spectra of the pure drug formulation (a), sitagliptin (b), and chitosan (c). 


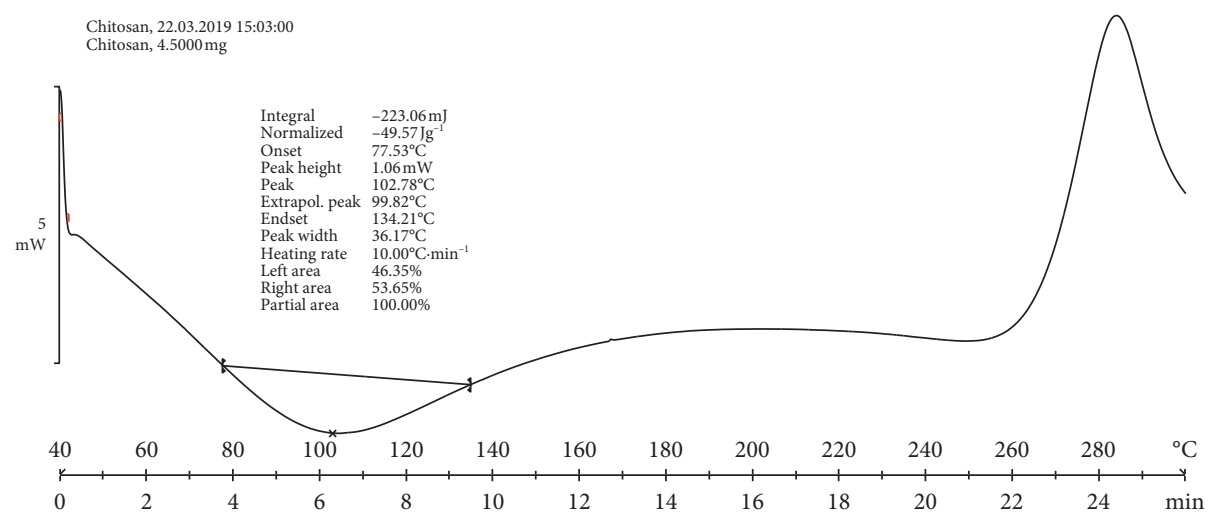

(a)

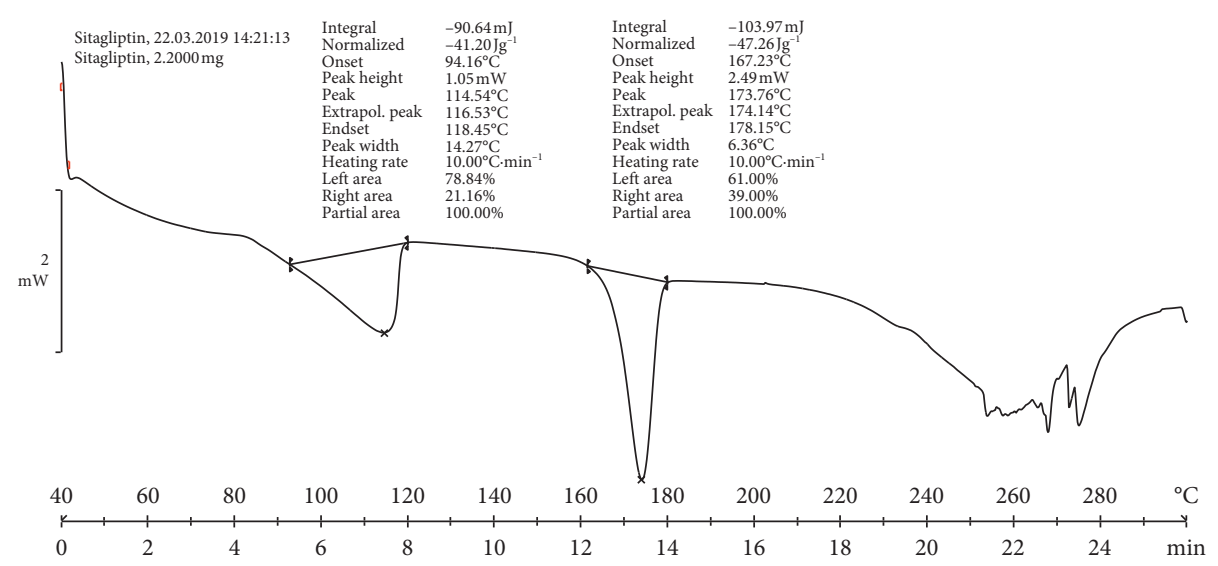

(b)

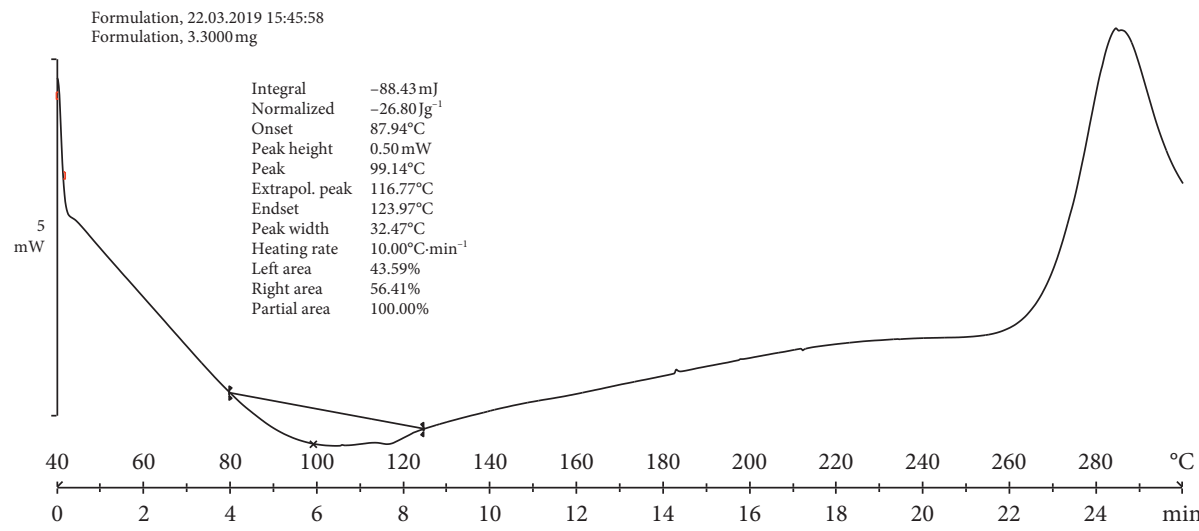

(c)

Figure 6: DSC analysis of the pure drug (sitagliptin (a), chitosan (b), and the formulation (c)).

The FTIR spectra of the pure drug showed significant bands at 3,339.06 (amine functional group), 3,149.80 (C-H stretching), and $1,633.71 \mathrm{~cm}^{-1}$ ( $\mathrm{C}=\mathrm{O}$ group); $\mathrm{N}-\mathrm{H}$ bending occurs around $1,597.06 \mathrm{~cm}^{-1}$ for primary amides, while for secondary amides it occurs around $1,514.12 \mathrm{~cm}^{-1}$. In terms of the major characteristic peaks, those for pure chitosan appeared around $1,141.75 \mathrm{~cm}^{-1}, 1,371.79 \mathrm{~cm}^{-1}$, and $1,315.41 \mathrm{~cm}^{-1}$, corresponding to the presence of $\mathrm{O}-\mathrm{H}$ bending, $\mathrm{C}-\mathrm{O}$ stretching, $\mathrm{C}-\mathrm{N}$ stretching, $\mathrm{C}-\mathrm{O}-\mathrm{H}$ bending $\left(1,415.75 \mathrm{~cm}^{-1}\right)$. Further, a significant band was observed at
$3,794.23 \mathrm{~cm}^{-1}$ for aromatic C-H stretching. Similar significant characteristic peaks of the drug remain unchanged in the formulation (Figure 5(a)), which confirms that there was no significant interaction between SCN (Figure 5(b)) and the polymer (Figure 5(c)).

The DSC thermograms of the endothermic peak onset temperatures for pure chitosan appeared at $102.78^{\circ} \mathrm{C}$, while the broadened peak endset temperature occurred at $134.21^{\circ} \mathrm{C}$ (Figure 6(a)). However, the physical mixture of chitosan and the drug exhibited a peak onset temperature 


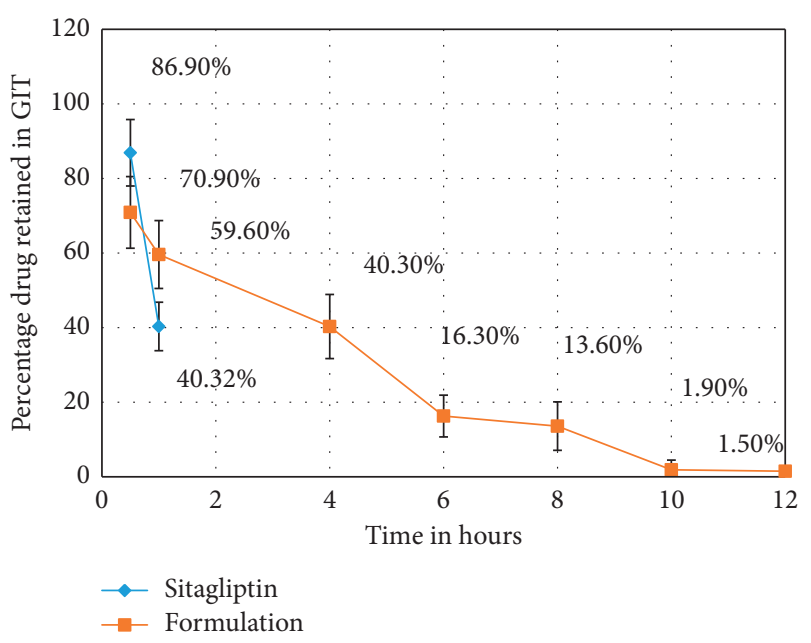

FIgURE 7: In vivo release comparisons in the stomachs of Sprague-Dawley rats following oral administration of the pure drug and nanoparticle formulation.

at $94.16^{\circ} \mathrm{C}$ and a peak endset temperature of $118.45^{\circ} \mathrm{C}$; the actual melting point of the drug had an onset peak temperature of $167.23^{\circ} \mathrm{C}$ and a peak endset temperature at $178.15^{\circ} \mathrm{C}$, respectively (Figure 6(b)). Further, an examination of the DSC analysis of the formulation showed that the drug peak disappeared given that the drug may have been in an amorphous state or a disordered crystalline state (Figure 6(c)).

Sprague-Dawley rats were orally administered SCN suspensions and SCN nanoparticles. The drug concentration percentage was read as previously described. At regular intervals of 0.5 hours, 1 hour, 4 hours, 6 hours, 8 hours, and 12 hours, the mucoadhesiveness of the particles was studied in vivo. The results revealed that the percent of SCN nanoparticles retained in the stomach was significantly higher than the percent of SCN suspension retained $(P<0.05)$, likely due to the narrow particle size of the prepared nanoparticles.

The polymer was deep inside the nanoparticles and had swollen into a large mass of polymers at a $\mathrm{pH}$ level of 1.2 (swelling behavior). As such, a large surface area had formed, and the interaction between the nanoparticle and mucin was conducive to prolonged residence in the gastrointestinal tract. Another reaction that favors adhesion is hydrogen bonding $[11,28]$. The chitosan polymers primarily adhered due to the hydrogen bond, the hydrophobic interactions with gastric mucin, the presence of amino groups, and electrostatic forces. The release percentage of SCN after 30 minutes was $29.1 \%$, while at 1 hour it was $40.1 \%$, at 4 hours it was $59.7 \%$, at 6 hours it was $83.7 \%$, at 8 hours it was $86.4 \%$, at 10 hours it was $98.1 \%$, and at 12 hours it was $98.4 \%$ (Figure 7). The percentage of the pure drug released after 30 minutes was around $40.32 \%$, and after 1 hour it was $86.9 \%$. Additionally, the SCN nanoparticles exhibited $29.1 \%$ drug release in 30 minutes, which means that a significant amount of SCN was weakly bound to or adsorbed on the surface of the nanoparticles. This is likely due to an "initial rapid release" and supports the notion that the formulation exhibits rapid and significant therapeutic effects on patients in clinical settings. When compared with the pure drug, the SCN nanoparticles showed a sustained release pattern.

\section{Conclusions}

Based on our results, we were able to conclude that a nanoparticle with mucoadhesive properties could be optimized, and this formulation was developed to ensure that SCN could remain in the stomach over a prolonged period of time. The nanoparticle-based mucoadhesive substances investigated here represent a novel form of drug administration that is ideal for patients with T2DM.

\section{Data Availability}

The data from the SEM, particle size, in vitro release, FTIR, DSC, swelling, and in vivo release studies that were used to support the findings of this study are included within the manuscript.

\section{Conflicts of Interest}

The authors declare that they have no conflicts of interest.

\section{Authors' Contributions}

Chandramouli Ramnarayanan and Anroop Nair contributed to data curation. Nagaraja SreeHarsha contributed to funding acquisition. Nagaraja SreeHarsha, Jagadeesh G. Hiremath, Bandar E. AlDhubiab, Katharigatta N. Venugopala, and Mahesh Attimarad contributed to the investigation. Nagaraja SreeHarsha, Jagadeesh G. Hiremath, Roopashree Satish, and Arshia Shariff contributed to the methodology. Roopashree Satish contributed resources. Bandar E. Al-Dhubiab and Chandramouli Ramnarayanan supervised the paper. Jagadeesh Hiremath and Katharigatta N. Venugopala validated the paper. Nagaraja SreeHarsha and Anroop Nair wrote the original draft of the paper. Nagaraja SreeHarsha wrote, reviewed, and edited the paper.

\section{Acknowledgments}

The authors thank the Deanship of Scientific Research, King Faisal University, Al-Ahsa, Saudi Arabia, for funding this project (grant number: 17122014). The authors wish to thank Mr. Tameem M. Alyahian and Mr. Naif Ali Alunssif, who aided in chemical procurement formulation preparation, and instrument handling. English language editing of this manuscript was provided by Journal Prep Services.

\section{References}

[1] M. Jahangir, S. Imam, and I. Kazmi, "Type 2 diabetes current and future medications: a short review," International Journal of Pharmaceutics \& Pharmacology, vol. 2017, no. 1, p. 101, 2017.

[2] N. Kheir, M. Zaidan, H. Younes, M. El Hajj, K. Wilbur, and P. J. Jewesson, "Pharmacy education and practice in 13 middle 
eastern countries," American Journal of Pharmaceutical Education, vol. 72, no. 6, p. 133, 2008.

[3] T. A. Elhadd, A. A. Al-Amoudi, and A. S. Alzahrani, "Epidemiology, clinical and complications profile of diabetes in Saudi Arabia: a review," Annals of Saudi Medicine, vol. 27, no. 4, pp. 241-250, 2007.

[4] A. A. Al-Khader, "Impact of diabetes in renal diseases in Saudi Arabia," Nephrology Dialysis Transplantation, vol. 16, no. 11, pp. 2132-2135, 2001.

[5] P. Aschner, "Recent advances in understanding/managing type 2 diabetes mellitus," F1000Research, vol. 6, p. 1922, 2017.

[6] H.-Q. Dong, M. Li, F. Zhu, F.-L. Liu, and J.-B. Huang, "Inhibitory potential of trilobatin from Lithocarpus polystachyus Rehd against $\alpha$-glucosidase and $\alpha$-amylase linked to type 2 diabetes," Food Chemistry, vol. 130, no. 2, pp. 261-266, 2012.

[7] M. Kikuchi, M. Haneda, D. Koya et al., "Efficacy and tolerability of vildagliptin as an add-on to glimepiride in Japanese patients with type 2 diabetes mellitus," Diabetes Research and Clinical Practice, vol. 89, no. 3, pp. 216-223, 2010.

[8] S. H. Nagaraja, B. E Al-Dhubiab, R. K. Tekade et al., "Novel preparation and effective delivery of mucoadeshive nanoparticles containing anti-diabetic drug," Indian Journal of Pharmaceutical Education and Research, vol. 53, no. 2s, pp. S43-S49, 2019.

[9] S. Desai and S. Bolton, "A floating controlled-release drug delivery system: in vitro-in vivo evaluation," Pharmaceutical Research, vol. 10, no. 9, pp. 1321-1325, 1993.

[10] G. A. Herman, A. Bergman, F. Liu et al., "Pharmacokinetics and pharmacodynamic effects of the oral DPP-4 inhibitor sitagliptin in middle-aged obese subjects," The Journal of Clinical Pharmacology, vol. 46, no. 8, pp. 876-886, 2006.

[11] S. Harsha, M. Attimard, T. A. Khan et al., "Design and formulation of mucoadhesive microspheres of sitagliptin," Journal of Microencapsulation, vol. 30, no. 3, pp. 257-264, 2013.

[12] S. Prasanthi, A. R. Prasad, Y. G. Kumar, R. N. Babu, M. Sudhir, and P. S. Babu, "Formulation and evaluation of sitagliptin phosphate and simvastatin bilayered tablets," Indo American Journal of Pharmaceutical Research, vol. 5, pp. 2231-6876, 2015.

[13] P. S. Maringanti and C. Nalagonda, "Formulation and evaluation of sitagliptin phosphate and metformin hydrochloride trilayered tablets," International Journal of Drug Delivery, vol. 5 , no. 1, p. 15, 2013.

[14] K. Keerthi, A. Kothamasu, S. N. Makineni, and K. K. Brahmandam, "Formulation and evaluation of sitagliptin phosphate gastro retentive tablets," Caribbean Journal of Science and Technology, vol. 2, pp. 270-281, 2014.

[15] A. Boutayeb and S. Boutayeb, "The burden of non communicable diseases in developing countries," International Journal for Equity in Health, vol. 4, no. 1, p. 2, 2005

[16] Y. M. K. Farag and M. R. Gaballa, "Diabesity: an overview of a rising epidemic," Nephrology Dialysis Transplantation, vol. 26, no. 1, pp. 28-35, 2010.

[17] M. Kikuchi, N. Abe, M. Kato, S. Terao, N. Mimori, and H. Tachibana, "Vildagliptin dose-dependently improves glycemic control in Japanese patients with type 2 diabetes mellitus," Diabetes Research and Clinical Practice, vol. 83, no. 2, pp. 233-240, 2009.

[18] S. A. A. Rizvi and A. M. Saleh, "Applications of nanoparticle systems in drug delivery technology," Saudi Pharmaceutical Journal, vol. 26, no. 1, pp. 64-70, 2018.

[19] W.-Z. Jiang, Y. Cai, and H.-Y. Li, “Chitosan-based spray-dried mucoadhesive microspheres for sustained oromucosal drug delivery," Powder Technology, vol. 312, pp. 124-132, 2017.
[20] T. M. Ways, W. Lau, and V. Khutoryanskiy, "Chitosan and its derivatives for application in mucoadhesive drug delivery systems," Polymers, vol. 10, no. 3, p. 267, 2018.

[21] A. Mitra and B. Dey, "Chitosan microspheres in novel drug delivery systems," Indian Journal of Pharmaceutical Sciences, vol. 73, no. 4, pp. 355-366, 2011.

[22] T. Ahmed and B. Aljaeid, "Preparation, characterization, and potential application of chitosan, chitosan derivatives, and chitosan metal nanoparticles in pharmaceutical drug delivery," Drug Design, Development and Therapy, vol. 10, pp. $483-507,2016$.

[23] K. V. Ranga Rao and P. Buri, "A novel in situ method to test polymers and coated microparticles for bioadhesion," International Journal of Pharmaceutics, vol. 52, no. 3, pp. 265-270, 1989.

[24] A. Haq Asif, S. Harsha, N. Hodalur Puttaswamy, and B. E. AlDhubiab, "An effective delivery system of sitagliptin using optimized mucoadhesive nanoparticles," Applied Sciences, vol. 8 , no. 6 , p. $861,2018$.

[25] J. J. Holst and C. F. Deacon, "Inhibition of the activity of dipeptidyl-peptidase IV as a treatment for type 2 diabetes," Diabetes, vol. 47, no. 11, pp. 1663-1670, 1998.

[26] J. Seewoodhary and S. C. Bain, "Novel treatments for type 2 diabetes," British Journal of General Practice, vol. 61, no. 586, pp. 325.6-326, 2011.

[27] G. Ponchel and J.-M. Irache, "Specific and non-specific bioadhesive particulate systems for oral delivery to the gastrointestinal tract," Advanced Drug Delivery Reviews, vol. 34, no. 2-3, pp. 191-219, 1998.

[28] N. S. Harsha and R. H. S. Rani, "Drug targeting to lungs by way of microspheres," Archives of Pharmacal Research, vol. 29 , no. 7 , pp. 598-604, 2006. 

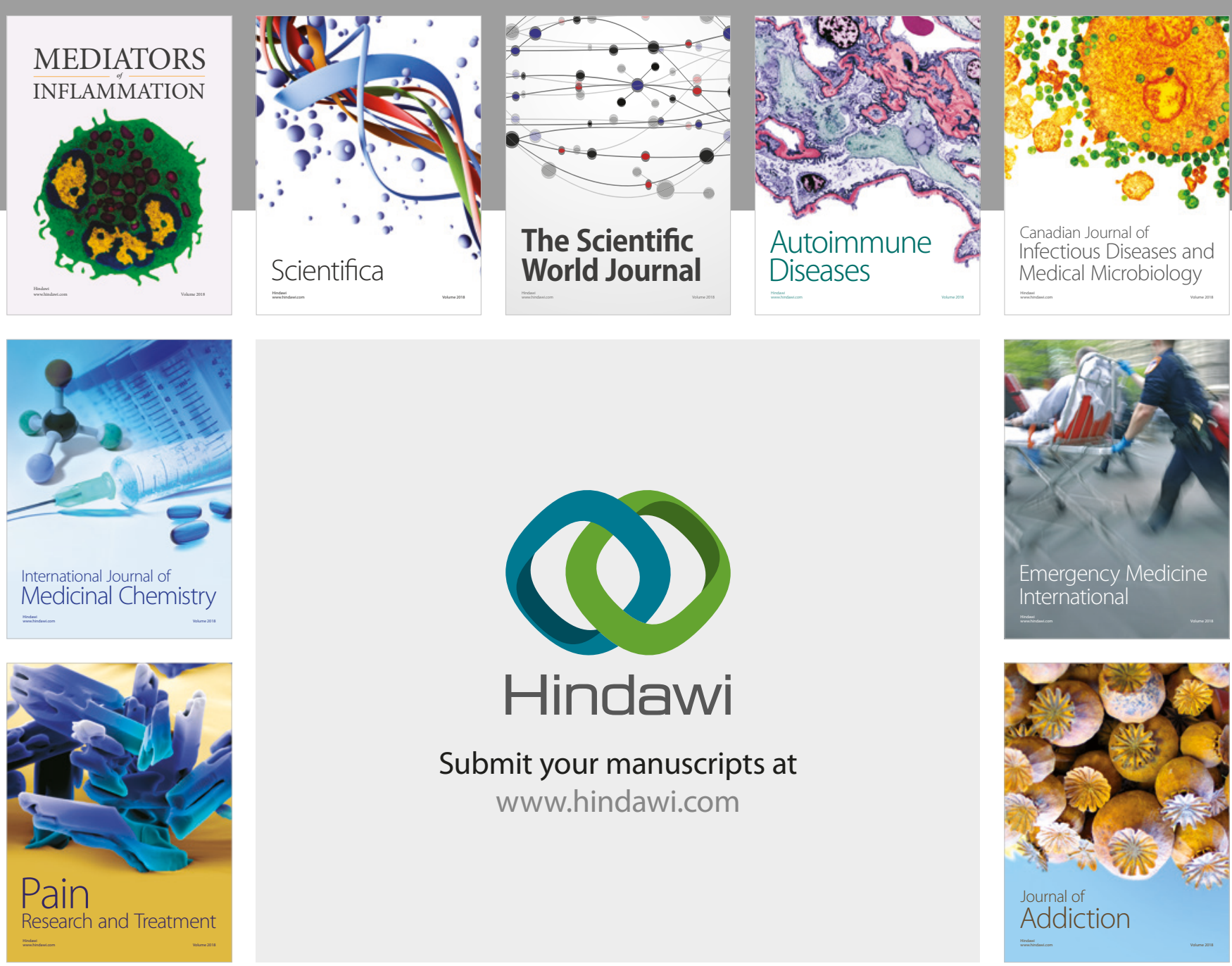

Canadian Journal of
Infectious Diseases and Medical Microbiology

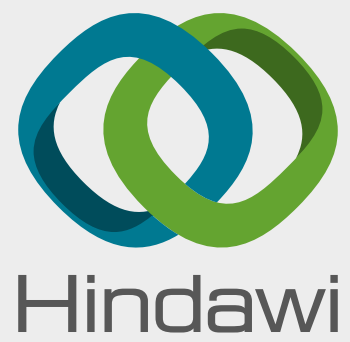

Submit your manuscripts at

www.hindawi.com
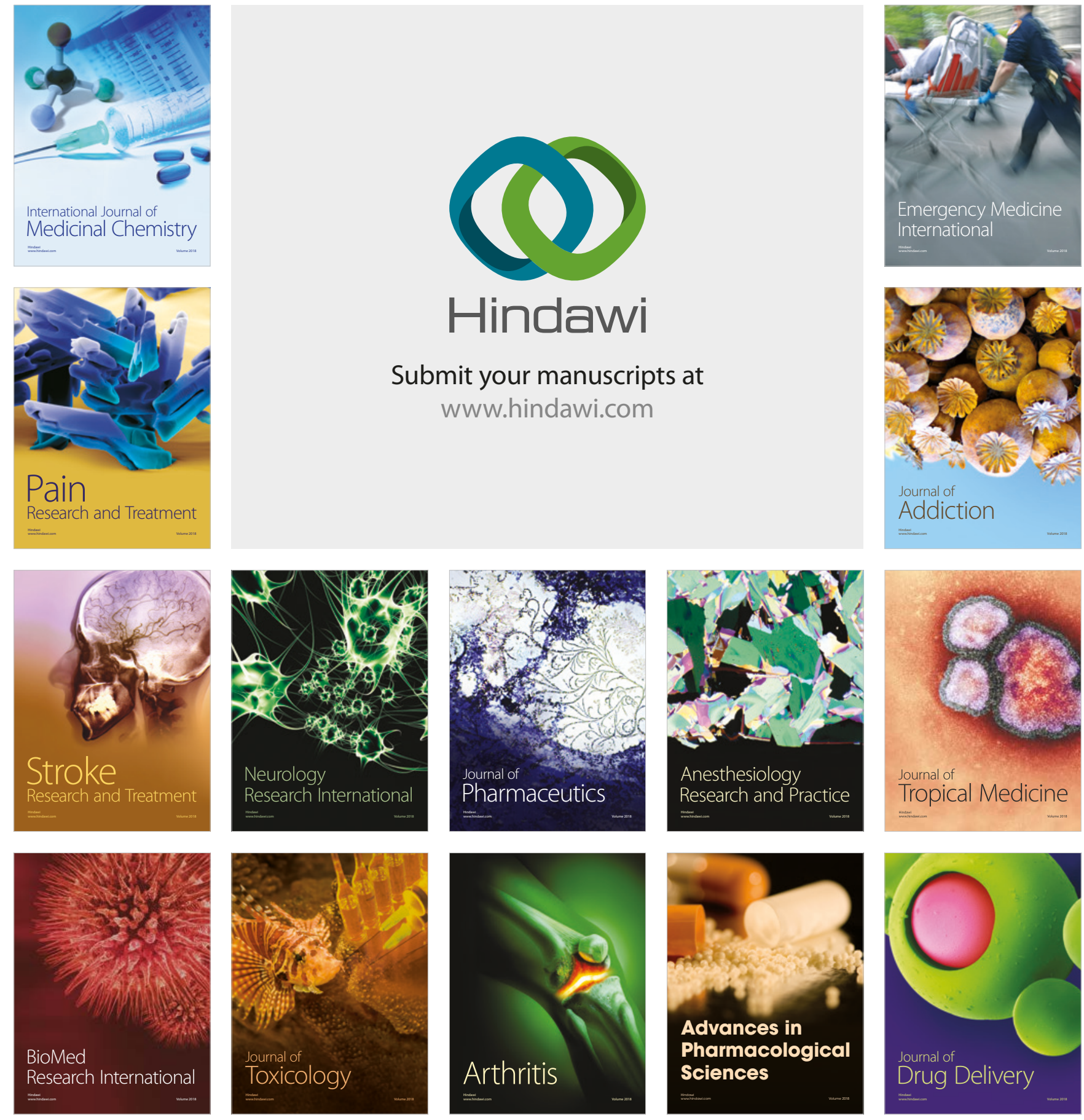